\title{
Spontaneous Coronary Artery Dissection and Takotsubo Cardiomyopathy: MRI Demonstration of the Underlying Mechanism
}

\author{
Cecchetto $A^{1}$, Dalla Chiara $E^{2}$, Cicciò $C^{2}$, Molon $G^{1}$, Dugo $C^{1}$, Barbieri $B^{1}$, Carbognin $G^{2}$, \\ Bonapace $\mathbf{S}^{1}$ \\ ${ }^{I}$ Cardiology Department, IRCCS, Sacro Cuore Don Calabria Hospital, Negrar, Verona, Italy \\ ${ }^{2}$ Radiology Department, IRCCS, Sacro Cuore Don Calabria Hospital, Negrar, Verona, Italy
}

*Corresponding Author: Antonella Cecchetto, Cardiology Department, IRCCS, Sacro Cuore Don Calabria Hospital, Negrar, Verona, Italy, Email: antonella.cecchetto@sacrocuore.it

\begin{abstract}
Spontaneous coronary artery dissection (SCAD) and Takotsubo cardiomyopathy (TTC) are two non-atherosclerotic causes of myocardial infarction. They share several common features and, in both, the exact mechanism has yet to be fully established. Hence, we want to do further consideration based on pathophysiology through cardiac magnetic resonance (CMR).
\end{abstract}

\section{TEXT}

In the literature, hypotheses have been formulated about the coexistence between SCAD and TTC, since one can determine the other. We briefly present two cases of SCAD of distal left anterior descending (LAD) coronary artery with CMR presentation similar to TTC. Two women of 53 and 54 years old came to the emergency room for chest pain, arising after a stressful event. ECG documented anterior ST segment elevation (Fig. 1A) and transthoracic echocardiogram showed left ventricle (LV) apex akinesia (Fig. 1L). The patients underwent to urgent coronary angiography (CA), that showed SCAD of LAD coronary artery (Fig. 1C-D). Apex and mid wall akinesia with basal segments hyperkinesia was visible, like TTC, at ventriculography (Fig. 1E). There was a little rise in Troponin I to laboratory tests and later ECG documented anterior $\mathrm{T}$ waves inversion (Fig. 1B). The patients underwent to CMR. We noted apex akinesia on cine sequences (Fig. $1 \mathrm{~F}$ $\mathrm{G})$, a large amount of oedema on the mid wall and apex of the LV on STIR sequences (Fig. 1 $\mathrm{H}-\mathrm{I}$ ) and no late enhancement (LE) after gadolinium injection (Fig.1 M-N-O).SCAD and TTC share clinical similarities: predilection for younger women, association with precipitating stressors, ischaemic electrocardiographic abnormalities, biomarker positivity and wall motion abnormalities that subsequently often normalize. In the literature, coexistence of SCAD and TTC has been reported in 14 patients. CMR was performed in 3 patients and
LE was present in the supply region of the dissected artery (1-2).

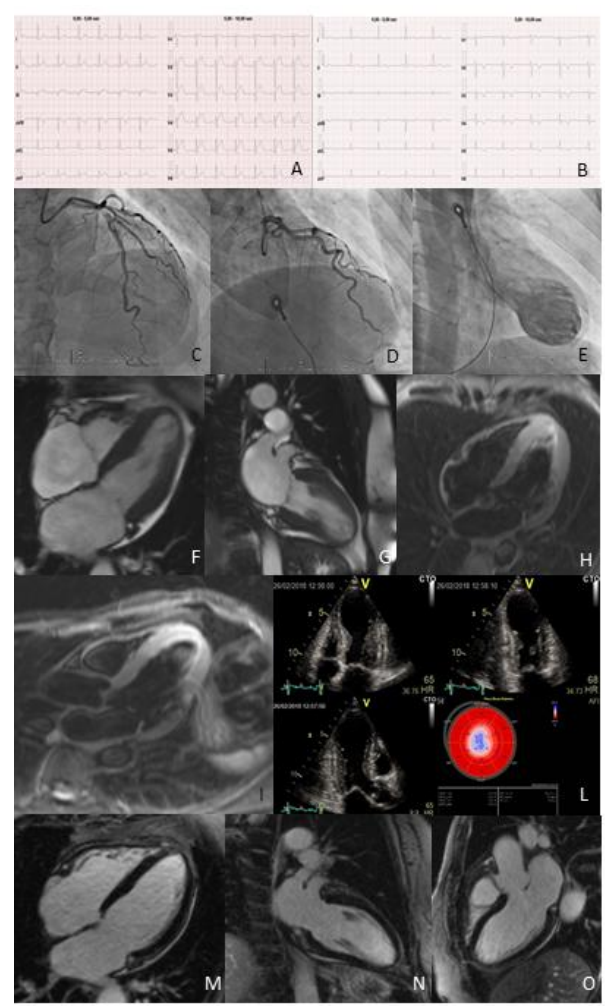

Figure1. ECG with anterior ST segment elevation, $A$; $E C G$ with anterior $T$ waves inversion, $B ; S C A D$ of $L A D$ coronary artery, $C-D$; ventriculography with apex and mid wall akinesia and basal segments hyperkinesia, E; apex akinesia on CMR cine sequences, F-G; oedema on the mid wall and apex of the LV on STIR sequences, H-I; echocardiogram 23- 4- chamber views and LV longitudinal strain bull eye, $L$; no LE after gadolinium injection, $M-N-O$. 
In our cases of SCAD with early reperfusion, release of Troponin was minimal with total recovery of cardiac function. Despite LAD artery was large and wrapped around the LV apex, we noted an extension of LV akinesia larger then the supply region of the dissected artery. Consequently, CMR presentation was similar to TTC, with a large amount of apical and mid wall oedema with absence of LE, demonstration of myocardial stunning rather than necrosis.

Alternatively, this could be the proof of the simultaneous presence of TTC and SCAD. SCAD of a well developed LAD could be the stressful event leading to TTC or mechanical solicitations associated with wall motion abnormalities in TTC could cause dissection of intima (3). In conclusion, CMR features of SCAD patients can be similar to TTC. Probably SCAD of a well developed LAD may result in apical wall motion abnormalities and oedema similar to TTC, therefore the angiographer should scrutinize the CA to rule out SCAD in all patients with clinical, echocardiographic and CMR presentation suspected for TTC. Alternatively, considering clinical similarities of both conditions, this could be the proof of the coexistence of TTC and SCAD in the same patient.

\section{REFERENCES}

[1] Buccheri D, Zambelli G. The link between spontaneous coronary artery dissection and Takotsubo cardiomyopathy: analysis of the published cases. J Thorac Dis. 2017; 9(12):5489-5492.

[2] Y-Hassan S, Henareh L. Spontaneous coronary artery dissection triggered post-ischemic myocardial stunning and Takotsubo syndrome: two different names for the same condition. Cardiovasc Revasc Med. 2013; 14:109-12.

[3] Y-Hassan S, Themudo R, Maret E. Spontaneous coronary artery dissection and takotsubo syndrome: the chicken or the egg causality dilemma. Catheter Cardiovasc Interv. 2017;89:1215-8.

Citation: Cecchetto A, Dalla Chiara E, Cicciò C, Molon G, Dugo C, Barbieri B, Carbognin G, Bonapace S. Spontaneous Coronary Artery Dissection and Takotsubo Cardiomyopathy: MRI Demonstration of the Underlying Mechanism.ARC Journal of Cardiology. 2018; 4(2) 13-14. doi: dx.doi.org/11.20431/24555991.0402004

Copyright: (C) 2018Authors. This is an open-access article distributed under the terms of the Creative Commons Attribution License, which permits unrestricted use, distribution, and reproduction in any medium, provided the original author and source are credited. 